\title{
Surrogate measures of insulin sensitivity vs the hyperinsulinaemic-euglycaemic clamp: a meta-analysis. Are there not some surrogate indexes lost in this story? Reply to Bastard JP, Rabasa-Lhoret R, Laville $M$ and Disse $E$ [letter]
}

\author{
Julia Otten • Bo Ahrén • Tommy Olsson
}

Received: 20 October 2014 / Accepted: 23 October 2014 / Published online: 22 November 2014

(C) Springer-Verlag Berlin Heidelberg 2014

Keywords Hyperinsulinaemic-euglycaemic clamp · Insulin resistance $\cdot$ Insulin sensitivity $\cdot$ Surrogate index

\section{Abbreviation \\ HEC Hyperinsulinaemic-euglycaemic clamp}

To the Editor: We agree with Dr Bastard and colleagues [1] that the variety of available tools provides an opportunity to assess different aspects of insulin sensitivity. In our extensive literature search on surrogate measures of insulin sensitivity, we found estimations of the correlation to the hyperinsulinaemiceuglycaemic clamp (HEC) for 31 indices based on fasting samples and 65 indices based on the OGTT [2].

The correlation strength between the accepted reference method, HEC, and a surrogate index is strongly influenced by the accuracy of the HEC, which varies depending on factors such as insulin dose and examination length. We therefore required a minimum of five papers for a given surrogate to be included in our review. However, the meta-analyses of the 76 surrogate measures that had been reported in fewer than five papers can be found in the electronic supplementary material linked to our original paper (ESM Table 2 for OGTT-based indices and ESM Table 3 for fasting indices) [2].

The SIisOGTT index reported by Bastard et al [1] is described in ESM Table 2 [2]. The meta-analysis for

J. Otten $(\bowtie) \cdot$ T. Olsson

Department of Public Health and Clinical Medicine, Medicine,

Umeå University, SE-90185 Umeå, Sweden

e-mail: julia.otten@medicin.umu.se

B. Ahrén

Department of Clinical Sciences Lund, Lund University,

Lund, Sweden
SIisOGTT was based on three articles [3-5] and resulted in a pooled correlation of $0.74(95 \%$ CI $0.60,0.83)$. The correlation strength of 0.74 was slightly higher than the other indices reported in detail in our paper. However, the confidence interval was wider compared with the Stumvoll MCR, Matsuda, Stumvoll ISI, Gutt and Revised QUICKI indices.

The meta-analysis for the Disse index based on two articles gave a pooled correlation of $r=0.73$ (ESM Table 3 ). The Avignon SiM index was reported in three papers, which amounted to a correlation of $r=0.70$ (ESM Table 2).

We aimed to rank the different surrogate measures found in the literature and to assess whether fasting indices can be used instead of the more time-consuming OGTT-based measures. According to our interpretation, the Revised QUICKI fasting surrogate index is as good as the OGTT-based Stumvoll MCR, OGIS, Matsuda, Stumvoll ISI and Gutt indices for estimating insulin sensitivity. However, we look forward to future studies that further examine the indices described in ESM Tables 2 and 3 in relation to the HEC.

Funding This work received no specific funding.

Duality of interest The authors declare that there is no duality of interest associated with this manuscript.

Contribution statement All authors were responsible for the conception and design of the manuscript, drafting the response letter and revising it critically for important intellectual content. All authors approved the version to be published.

\section{References}

1. Bastard JP, Rabasa-Lhoret R, Laville M, Disse E (2014) Surrogate measures of insulin sensitivity vs the hyperinsulinaemic-euglycaemic clamp: a meta-analysis. Are there not some surrogate indexes lost in this story? Diabetologia doi 10.1007/s00125-014-3424-4 
2. Otten J, Ahrén B, Olsson T (2014) Surrogate measures of insulin sensitivity vs the hyperinsulinaemic-euglycaemic clamp: a metaanalysis. Diabetologia 57:1781-1788

3. Lorenzo C, Haffner SM, Stancakova A, Laakso M (2010) Relation of direct and surrogate measures of insulin resistance to cardiovascular risk factors in nondiabetic Finnish offspring of type 2 diabetic individuals. J Clin Endocrinol Metab 95: $5082-5090$
4. Pigeon E, Riou ME, St-Onge J et al (2009) Validation of a simple index (SIisOGTT) of insulin sensitivity in a population of sedentary men. Diabetes Metab 35:398-403

5. Bastard JP, Lavoie ME, Messier V, Prud'homme D, Rabasa-Lhoret R (2012) Evaluation of two new surrogate indices including parameters not using insulin to assess insulin sensitivity/resistance in nondiabetic postmenopausal women: a MONET group study. Diabetes Metab 38:258-263 